Atomistic Simulations of Grain
Boundary Pinning in CuFe Alloys

L. A. Zepeda-Ruiz, G. H. Gilmer, B. Sadigh, J. A. Caro, T. Oppelstrup

June 1, 2005

Applied Physics Letters 
This document was prepared as an account of work sponsored by an agency of the United States Government. Neither the United States Government nor the University of California nor any of their employees, makes any warranty, express or implied, or assumes any legal liability or responsibility for the accuracy, completeness, or usefulness of any information, apparatus, product, or process disclosed, or represents that its use would not infringe privately owned rights. Reference herein to any specific commercial product, process, or service by trade name, trademark, manufacturer, or otherwise, does not necessarily constitute or imply its endorsement, recommendation, or favoring by the United States Government or the University of California. The views and opinions of authors expressed herein do not necessarily state or reflect those of the United States Government or the University of California, and shall not be used for advertising or product endorsement purposes. 


\title{
Atomistic simulations of grain boundary pinning in CuFe alloys
}

\author{
L. A. Zepeda-Ruiz, ${ }^{1, *}$ G. H. Gilmer, ${ }^{1}$ B. Sadigh, ${ }^{1}$ A. Caro,${ }^{1}$ and T. Oppelstrup ${ }^{1,2}$ \\ ${ }^{1}$ Chemistry and Materials Science Directorate, \\ Lawrence Livermore National Laboratory, Livermore, CA 94550 \\ ${ }^{2}$ NADA, Royal Institute of Technology (KTH), 10044 Stockholm, Sweden
}

(Dated: May 31, 2005)

\begin{abstract}
We apply a hybrid Monte Carlo-molecular dynamics code to the study of grain boundary motion upon annealing of pure $\mathrm{Cu}$ and $\mathrm{Cu}$ with low concentrations of Fe. The hybrid simulations account for segregation and precipitation of the low solubility Fe, together with curvature driven grain boundary motion. Grain boundaries in two different systems, a $\Sigma 7+$ U-shaped half-loop grain and a nanocrystalline sample, were found to be pinned in the presence of Fe concentrations exceeding $3 \%$.
\end{abstract}

PACS numbers: Valid PACS appear here

*Electronic address: zepedaruiz1@llnl.gov 


\section{INTRODUCTION}

The stabilization of grain boundaries in nanocrystalline materials has received much attention in recent years [1-5]. The Hall-Petch relation predicts that the yield strength or hardness of polycrystalline materials increases with decreasing grain size, due to inhibited dislocation motion. But the possibility of taking full advantage of restricted dislocation motion in these materials seems to be limited to grain sizes above a critical size (10-30 nm); below this value, the strength decreases with decreasing grain size. Grain sliding or other types of grain boundary accommodation are thought to predominate in this regime. For this reason we apply atomistic modeling to study the inhibition of grain boundary motion by impurity drag or precipitate pinning mechanisms. The ability to stabilize the boundaries in this way may extend the region of normal Hall-Petch behavior to smaller grain sizes, and allow the fabrication of materials with greater hardness.

The early models of impurity drag are based on continuum models of grain boundary structure that tacitly assume either a diffuse interface, which can "drag" an impurity cloud along because of a lower potential energy of the impurities in the interfacial region, or a mechanism analogous to impurity drag due to a Cottrell atmosphere of impurities around a dislocation [6, 7]. Impurity drag is the result of attractive forces resulting from the stress field surrounding a dislocation. Neither of these models is very useful, since the diffuse interface description is not consistent with the fact that the actual grain boundary "thickness" is only a few lattice spacings, and the stress field surrounding the grain boundary has a very short range, much less than that of a dislocation [8]. Atomic interactions and configurations are essential for accurate modeling. Atomistic models have shown clearly that the mismatch of the two orientations produces sites at the grain boundary that are in strong tensile stress, and others in compressive stress, and that these highly localized sites are usually the major players in the binding of impurity atoms [9]. For this reason we have applied molecular dynamics (MD) and Monte Carlo (MC) techniques to this study, since the atomic structure and resulting local stresses are then fully represented.

In this paper, we will use MD simulations to measure curvature-driven grain boundary migration for systems with different concentrations of Fe. The initial distribution of the impurities is an important issue for this study, since the time scale feasible for MD simulations is relatively short, on the order of nanoseconds, and solid-state diffusion of Fe atoms is 
quite small. (For the same reason, MD simulations of other slow processes such as creep are difficult.) The fabrication of nanocrystalline materials occurs on a much longer time scale and usually involves either deposition, with rapid interfacial and/or surface diffusion, or high temperature deformations. Both methods involve high mobility and are likely to allow equilibration of impurities between the bulk and grain boundaries. Therefore, we start our simulations with an equilibration run based on the Metropolis MC algorithm that produces the initial distribution of impurities.

\section{COMPUTATIONAL METHOD}

In order to describe alloy properties, two major issues have to be addressed. One is the adequacy of the potentials to describe the materials properties, and the second is to develop the right tools to prepare the samples in representative initial states, in particular addressing the grain boundary segregation issue. For the first point, we have analyzed in detail the properties of the $\mathrm{Cu}-\mathrm{Fe}$ potentials available in the literature $[10,11]$ and found that the Ludwig et al. potential [12] reproduces the measured phase diagram most accurately. For the second point, we have developed a massively parallel MC-MD code in the transmutation ensemble. This hybrid code performs MC events and MD time steps based on the same system of atoms: computational cell, interatomic potential, boundary conditions, etc. It includes a sequence of static transmutations using the Metropolis algorithm, followed by MD steps to relax the system. We found that cycles of transmutation trials on $50 \%$ of the atoms, followed by $100 \mathrm{MD}$ steps (both processes at the same temperature) adequately equilibrate the system for a given difference in chemical potential. In this way the equilibrium concentrations in the grain boundary and bulk are obtained and we will refer to this process as an MC-MD run. Details of the code will be published elsewhere.

Two samples with different microstructures were used in our study as shown in Figure 1. The first one, a nanocrystalline sample, was created using a Voronoi construction, with random location and orientation of the grains. It consists of 5 grains with a mean size of $5 \mathrm{~nm}$ and has dimensions of $40 \mathrm{a}_{0} \times 40 \mathrm{a}_{0} \times 40 \mathrm{a}_{0}$, where $\mathrm{a}_{0}$ is the $\mathrm{Cu}$ lattice constant and contains approximately 256000 atoms. Periodic boundary conditions are used in the 3 directions. The color scheme for the atoms indicates the crystallographic orientation of the grains. The second sample, a U-shaped half-loop bicrystal is created by rotating grain 
2 through $38.2^{\circ}$ about the $z$-direction (a common $\langle 111\rangle$ direction) [13-15]. This value of rotation corresponds to high symmetry $\Sigma 7$ grain boundaries along the straight branches shown in Fig. 1(b). These boundaries provide the driving force for motion. The average mobility of the curved segment is measured during annealing. The dimensions are $17 \sqrt{6} \mathrm{a}_{0} \times$ $48 \sqrt{2} \mathrm{a}_{0} \times 6 \sqrt{3} \mathrm{a}_{0}$ (approximately 110000 atoms) along $x, y$, and $z$, respectively $(x, y$, and $z$ correspond to $\langle 112\rangle,\langle 110\rangle$, and $\langle 111\rangle$, respectively). Periodic boundary conditions are used in the $x$ and $z$ directions and free surfaces in the $y$ direction. A layer of static atoms is introduced at the left surface in the $y$ direction to avoid grain rotation. In both cases the simulations are performed in an NVT ensemble after a short thermalization and volume relaxation under 0 pressure.

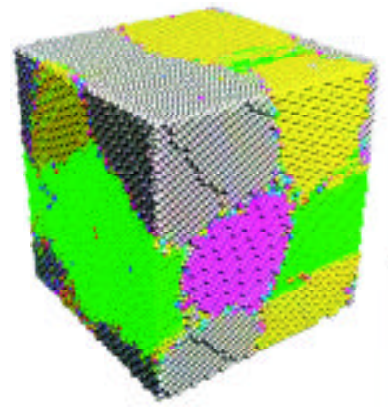

(a)

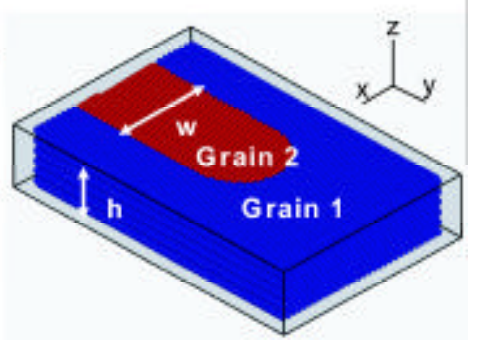

(b)

FIG. 1: Nanocrystalline and half-loop samples.

\section{SIMULATIONS OF GRAIN BOUNDARY PINNING}

\section{A. Nanoycrystalline CuFe}

We next consider the annealing of nanocrystalline samples with various concentrations of $\mathrm{Fe}$, namely $0 \%, 0.6 \%$, and $3 \%$. After loading the samples with Fe and relaxing them at the chosen annealing temperature of $950 \mathrm{~K}$, MD runs of 100 picosecond annealing were performed. A representative configuration with $3 \%$ Fe is shown in Fig. 2. It is clear that the majority of the Fe impurities (shown in red) is located in the grain boundaries, and small precipitates are formed.

Figure 3 shows cross sections of the $\mathrm{Cu}$ samples before and after the MD annealing run. The same initial configuration, shown in Fig. 3(a), was used for different concentrations of 


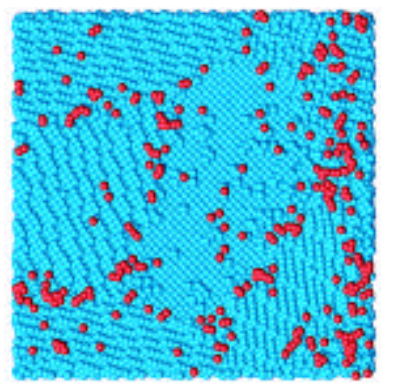

FIG. 2: Configuration obtained after loading $3 \%$ Fe as described above.

Fe. Essentially the same initial atomic positions are used for all cases, since the MC-MD runs cause only small atomic displacements. Since the same color scheme shown in Fig. 1 is used here, color indicates orientation alone, and Fe atoms are not differentiated from $\mathrm{Cu}$ atoms. It is clear that during annealing grain boundaries in pure $\mathrm{Cu}$ exhibit considerable migration, as shown by comparing Figs. 3(a) and (b). The shrinkage of the central grain and the growth of its neighbors is clearly visible. Smaller grain boundary displacements are observed when the system contains $0.6 \%$ Fe, as shown in Fig. 3(c). It is observed that while a small amount of Fe $(0.6 \%)$ is not sufficient to stop grain boundary migration, the sample with $3 \%$ Fe exhibits very small displacements for all grains. This result shows that impurities that segregate to the boundaries are able to stabilize the grain structure during annealing.

\section{B. Half-loop Grains}

Our grain boundary studies on nanocrystalline samples provide an indication of the mobility of the grain boundaries as a function of Fe concentration. As in experiments on nanocrystalline samples, it is difficult to extract precise mobilities from these results, since they are derived from grains with various crystallographic parameters and a distribution of sizes that change continuously during annealing. A solution to this problem is to study such properties using individual, well defined, grain boundaries. The U-shaped half-loop geometry shown in Fig. 1(b) has been used both experimentally and computationally to extract individual properties of a variety of grain boundaries [13-15]. This geometry is ideal for mobility studies since the driving force and the shape of the moving boundary segment remain constant during annealing. 

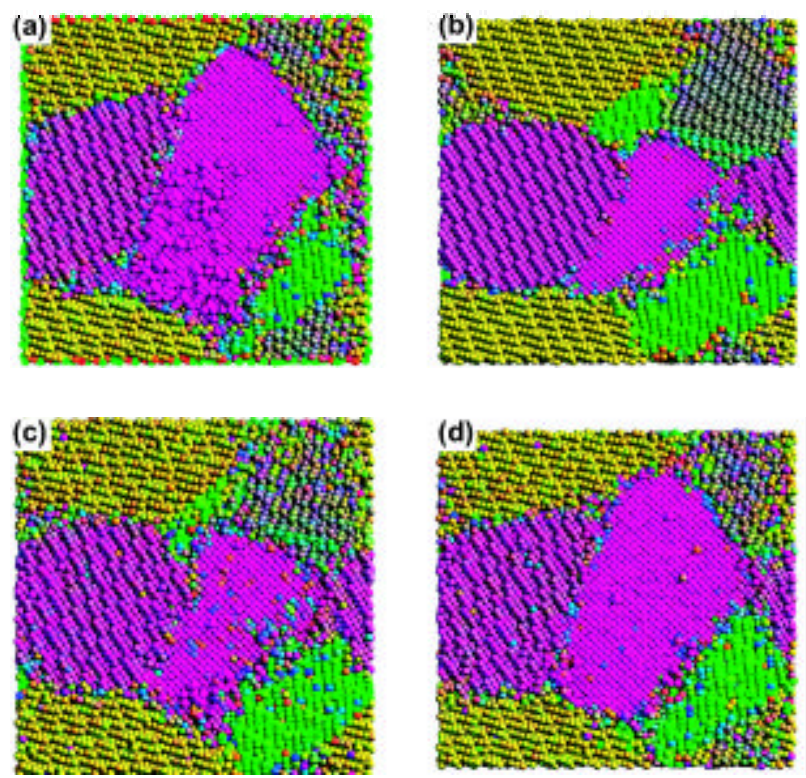

FIG. 3: Cross sections of the nanocrystalline samples. (a) represent the initial configuration for 3 different cases: (b), (c) and (d). The resulting configuration after 100 ps anneal at $960 \mathrm{~K}$ are shown in (b) for pure $\mathrm{Cu}$, (c) for $0.6 \% \mathrm{Fe}$ and (d) for $3 \% \mathrm{Fe}$.

Figure 4, shows a $\langle 111\rangle$ projection of the initial (a) and final configuration (b) after a 350 ps MD run of a $\Sigma 7$ grain boundary (straight segments) plus the half loop in pure $\mathrm{Cu}$ at $\mathrm{T}=667 \mathrm{~K}$. Only atoms with potential energies $100 \mathrm{meV}$ higher than the perfect crystal value are shown to mark the location of the grain boundary. After an initial transient, where the grain relaxes from its initial configuration, a steady-state migration is observed: the inner grain shrinks by the translation of the curved segment. The driving force, which is the $\Sigma 7$ grain boundary energy per unit area, remains constant during anneal.

Figure 5 shows in turn the initial (a) and final (b) configurations of a sample loaded with $0.91 \%$ Fe using the MC-MD run. Although this concentration value is below the solubility limit for this potential [11], we can observe a weak segregation of Fe (depicted in red in the figure) at the grain boundary. It can also be observed that there is no impurity drag under these conditions, but depinning from the initial segregated Fe atoms occurs.

By monitoring the distance traveled by the half-loop during MD annealing we can obtain a measure of the grain boundary mobility in the presence of Fe impurities. Figure 6 shows an Arrhenius plot of the displacements for the three samples studied. A substantial variation of the activation energy with impurity content is clearly observed: $0.06 \mathrm{eV}$ for pure $\mathrm{Cu}, 0.56$ 

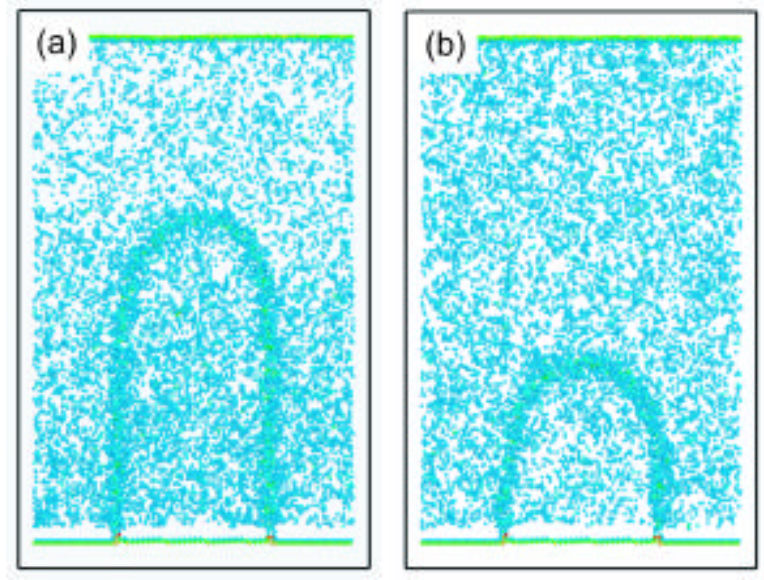

FIG. 4: Snapshots of (a) initial and (b) final configuration of pure $\mathrm{Cu} \Sigma 7$ half-loop grain boundary annealed at a temperature of $667 \mathrm{~K}$ for $350 \mathrm{ps}$.
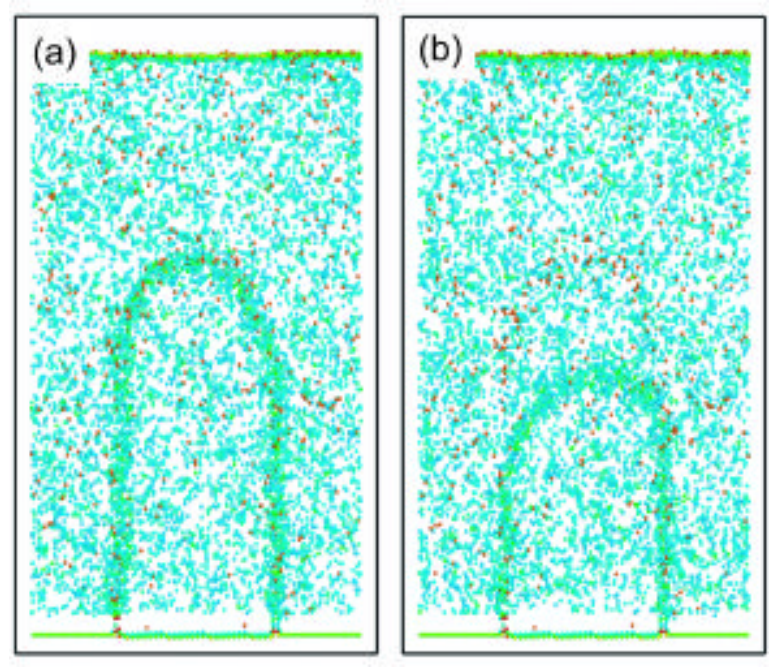

FIG. 5: Snapshots of (a) initial and (b) final configuration of $\mathrm{Cu} \Sigma 7$ half-loop grain boundary with $0.91 \%$ Fe annealed at a temperature of $1000 \mathrm{~K}$ for $350 \mathrm{ps.}$

$\mathrm{eV}$ for $\mathrm{Cu}-0.34 \% \mathrm{Fe}$, and $0.97 \mathrm{eV}$ for $\mathrm{Cu}-0.91 \%$ Fe. While the activation energy for grain boundary mobility for pure $\mathrm{Cu}$ is well below reported experimental measurements, less than $1 \%$ Fe changes it considerably, putting it in the range of experimental observations $[16,17]$. This result highlights the strong effect of impurities on mobility, even at low concentrations.

These measurements of grain boundary motion in the presence of impurities involve an average over a period of time where the grain boundary is pinned, and a time of fast motion 


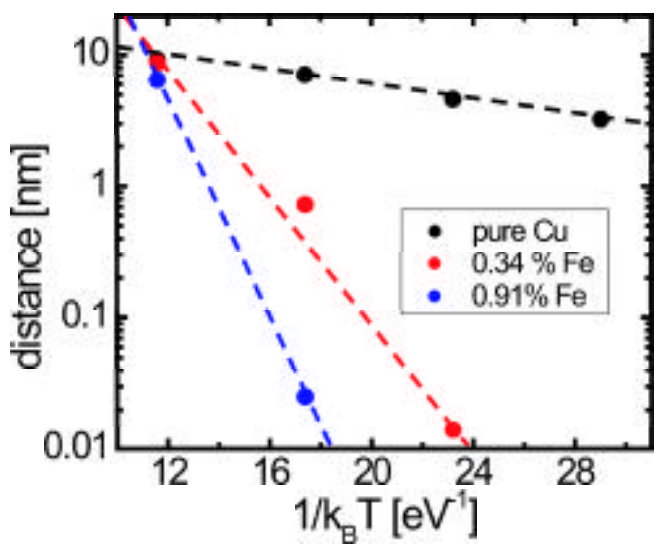

FIG. 6: Arrhenius plots of mobility for half-loop boundaries with different impurity concentrations measured from configurations represented by Figs. 4 and 5 .

until it meets new pinning points. Our geometry in these simulations is oversimplified but since the displacements correspond approximately to the grain diameter it provides a good estimate of the average speed in actual polycrystalline samples. In both cases, the activation energy is dominated by depinning.

\section{CONCLUSIONS}

In summary, we have simulated curvature driven grain boundary motion using a hybrid Monte Carlo-molecular dynamics simulator. In this way, we obtain initial conditions of polycrystalline samples with segregation of impurities to the grain boundaries. We find that Fe additions stabilize grain boundary motion during annealing, both in a configuration that includes a diversity of grains and in a configuration that provides a measurement of grain boundary mobility. Our simulations show that small concentrations of low solubility impurities are capable of causing drastic reductions in grain boundary mobilities. The presence of $1 \%$ Fe caused an order of magnitude increase in the Arrhenius barrier for grain boundary motion, and 3\% Fe essentially immobilized grain boundaries in our nanocrystalline sample. Our newly developed tools allow us to explore the important process of impurity segregation at grain boundaries and its effect on pinning, opening the possibility of modeling and designing extremely hard materials. 


\section{Acknowledgments}

We thank A. V. Hamza for insightful discussions and support. This work was performed under the auspices of the U.S. Department of Energy by the University of California, Lawrence Livermore National Laboratory under Contract No. W-7405-Eng-48.

[1] R. Siegl, M. Yan, and V. Vitek, Modelling Simul. Mater. Sci. Eng. 5, 105 (1997).

[2] J. Schiøtz and K. W. Jacobsen, Science 301, 1357 (2003).

[3] V. Yamakov, D. Wolf, S. R. Phillpot, A. K. Mukherjee, and H. Gleiter, Nature Mater. 3, 43 (2004).

[4] G. Duscher, M. F. Chisholm, U. Alber, and M. Rühle, Nature Mater. 3, 621 (2004).

[5] R. Schweinfest, A. T. Paxton, and M. W. Finnis, Nature 432, 1008 (2004).

[6] K. Lucke and K. Detert, Acta Met. 5, 638 (1957).

[7] J. W. Cahn, Acta Met. 10, 789 (1962).

[8] D. N. Seidman, 32, 235 (2002).

[9] X. Y. Liu, W. Xu, S. M. Foiles, and J. B. Adams, Appl. Phys. Lett. 72, 1578 (1998).

[10] A. Caro, P. E. A. Turchi, M. Caro, and E. M. Lopasso, J. Nucl. Mater. 336, 233 (2005).

[11] A. Caro, M. Caro, E. M. Lopasso, P. E. A. Turchi, and D. Farkas, submitted to J. Nucl. Mater.

[12] M. Ludwig, D. Farkas, D. Pedraza, and S. Schmauder, Modelling Simul. Mater. Sci. Eng. 6, 19 (1998).

[13] V. Y. Aristov, V. E. Fradkov, and L. S. Shvindlerman, Sov. Phys. Solid State 22, 1817 (1980).

[14] M. Upmanyu, R. W. Smith, and D. J. Srolovitz, Interface Sci. 6, 41 (1998).

[15] H. Zhang, M. Upmanyu, and D. J. Srolovitz, Acta Mater. 53, 79 (2005).

[16] B. Günther, A. Kumpmann, and H. D. Kunza, Scripta Met. Mater. 27, 833 (1992).

[17] R. Dannenberg, E. A. Stach, J. R. Groza, and B. J. Dresser, Thin Solid Films 370, 54 (2000). 